\title{
A NEW EFFECT-BASED CLASSIFICATION OF LASER APPLICATIONS IN SURGERY AND MEDICINE
}

\author{
Toshio Ohshiro \\ Japan Medical Laser Laboratory, Shinanomachi, Tokyo, Japan.
}

\begin{abstract}
In the last decade the applications of the laser in surgery and medicine have increased dramatically. With the increase of indications has come a concomitant increase in possible classification of laser reactions. The author presents a classification which is based on the laser/tissue reaction rather than on the hardware used to produce the laser beam. Laser/tissue reactions fall into two broad groups. When the tissue reaction to absorption of the incident laser energy results in photodestruction of, or an irreversible photomodulated change to the tissue architecture, then the level of reaction is higher than the survival threshold of the target cells. The author refers to this as high reactive-level laser treatment (HLLT), or more generally as laser surgery. On the other hand, the level of tissue reactivity to very low incident power and energy densities is well below the cells' survival threshold so that instead of being damaged the cells are directly activated by the low incident photon density. In this case the changes in the irradiated tissue are photoactivative and reversible: the author refers to this group of reactions as low reactive-level laser therapy (LLLT), or more generally as laser therapy. Both of these groups can be classed under the general heading of laser treatment (LT). LT is further subdivided into three main types: mono-type LT (Mo-LT, single laser treatment; multi-type LT (Mu-LT, multi-laser treatment); and concomitant LT (Cc-LT), in which any of the above LT types can be used in combination with conventional treatment methods. Mo-LT in turn contains pure LT (Pu-LT) single laser, single reaction; and auto-simultaneous LT (ASi-LT), single laser with a range of reaction types, each of which has its own abbreviation. Mu-LT contains two main sub-types, combined LT (Cb-LT) and compound LT (Cp-LT). Cb-LT concerns the same disease whereas Cp-LT is used to describe 2 or more diseases treated with the same or different lasers. Both $\mathrm{Cb}-\mathrm{LT}$ and $\mathrm{Cp}-\mathrm{LT}$ are further subclassified into the homogeneous and xenogeneous types, referring to the use of the same or different types of laser, respectively. If the lasers are applied at the same time, that is homo- or xeno-simultaneous laser treatment (HoSi-LT, XeSi-LT), and if at different times the term is homo- or xeno-succesive laser treatment (HoSu-LT, XeSu-LT). The various sub-sets of Mono-type and Multi-type laser treatment are further expanded, to give an accurate, treatment-based categorization of laser treatment. In addition to the above classification, the author has devised a graphical representation of laser surgical and therapeutic beams whereby the laser type, parameters, penetration depth, and tissue reaction can all be shown in a single illustration, which the author has termed the 'Laser Apple', due to the typical pattern generated when a laser beam is incident on tissue. Apple types fall into two main subdivisions, destructive or D-Apples (HLLT) which include the C-Apple (carbonization), V-Apple (vaporization) and so on, and the activative or A-Apple type (LLLT). When the above classification is combined and illustrated with the appropriate laser apple type or types, the author feels this offers an accurate and simple method of classifying laser/tissue reactions by the reaction, rather than by the laser used to produce the reaction.
\end{abstract}

Key words: Laser classification, laser therapy, laser surgery, LLLT, HLLT, photodestruction, photoactivation, Laser Apple

\section{Introduction}

The author has already presented his general classification of laser/tissue reactions into high-reactive-level laser treatment (HLLT, laser surgery) and low reactive-level laser therapy (LLLT, laser therapy), ${ }^{(1-3)}$ where the 'level' in these terms represents the level of reaction of the target cells to the incident laser energy and has really not a lot to do with the laser output power. In

Addressee for Correspondence:

Toshio Ohshiro MD DMSc,

Japan Medical Laser Laboratory, Shinanomachi JR Station Bldg., 2nd Floor, 34 Shinanomachi, Shimjuku, Tokyo, Japan 160.
HLLT the level of reaction is high, resulting in photodestruction and irreversible, mostly photothermally-mediated changes to the tissue architecture ranging from carbonization to protein denaturation. In LLLT the level of reaction is low, below the damage threshold of the target cells, and results in photobioactivation of the target cells and tissue to produce a range of beneficial therapeutic effects including amongst others enhanced wound healing, pain attenuation and accelerated repair to soft and hard tissue injuries. Photobioactivation can be used on its own, as in

Manuscript received: October 1996

Accepted for publication: December 1996 


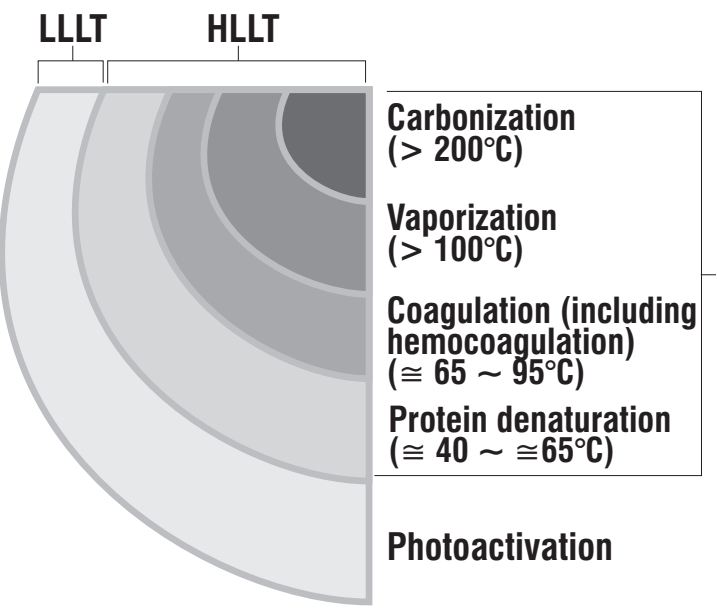

Fig 1: Range of photothermal and nonthermal bioreactions generated by a laser beam when absorbed in target tissue, showing the classification of bioreactions into photodestructive reactions (laser surgical indications) and photoactivative reactions (laser therapeutical reactions). Note that the outer photoactivative zones occur almost simultaneously with the photodestructive reactions.

laser therapy, or as a simultaneous effect which occurs along with the desired surgical effect. The above range of reactions associated with the overall concept of laser treatment (LT) are illustrated in Figure 1, and will form the basis for all of the classification arguments which follow. The author feels that these general classifications are too broad, and so in the present study presents a more detailed classification based on subdivisions of the main classifications of laser surgery and laser therapy which depend on a combination of factors such as the number of diseases being treated, the combination of lasers used and the class of the reaction. The author's has based many of the illustrations on his previously presented graphical representation of surgical and therapeutic lasers, the Laser Apple group, ${ }^{(4)}$ by which the scattering pattern and penetration depth of the beam is easily understood through a single illustration, in addition to full information on the laser type and the beam parameters. In vivo and in vitro experiments have provided visual corroboration of the author's suggested nomenclature. ${ }^{(5,6)}$ Figure 2 shows an example of a typical laser apple, in this case a $\mathrm{CO}_{2}$ laser $\mathrm{V}$-Apple (vaporization). ${ }^{4}$ From this illustration, the reader can see at a glance the laser type, the wavelength, the laser output power, the irradiated area and the irradiation time, in addition to the range of reactions and the approximate penetration depth.

\section{Laser Classification}

Under Ohshiro's system, laser treatment falls into three main classifications: mono-type laser treatment (Mo-LT), multi-type laser treatment (Mu-LT) and con-

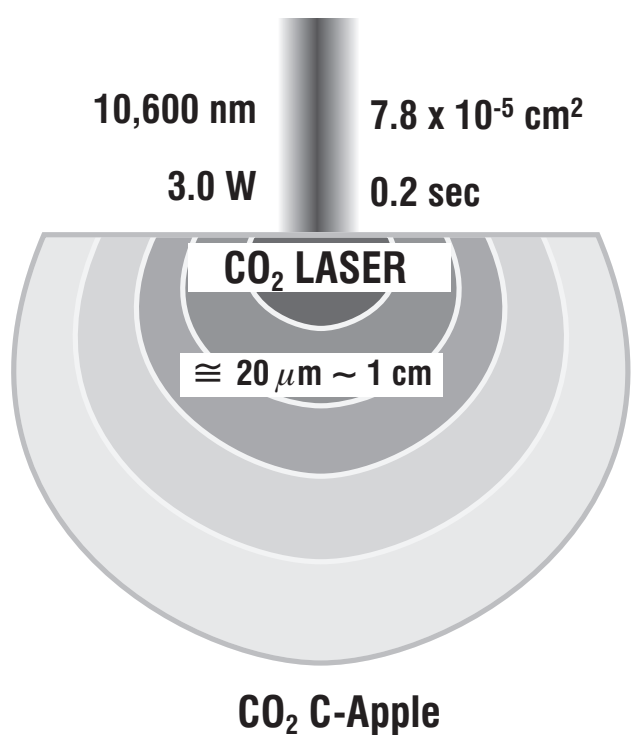

Fig 2: Typical laser apple, a $\mathrm{CO}_{2}$ laser $\mathrm{C}$-Apple, showing all relevant information including the laser type, wavelength, output power, irradiated area and exposure time from which the incident power and energy densities can be calculated. Additionally the reaction range and approximate penetration depth is given. All this information is thus displayed in a single simple schematic (from ref. 4: used with permission).

comitant-type laser treatment (Cc-LT) (Table 1). Monoand multi-type LT are further subclassified, and these will now be explained in detail.

\section{1: Mono-type Laser Treatment (Mo-LT)}

As the name suggests, Mo-LT is used to describe the use of a single laser, either in laser therapy (LLLT) laser surgery (HLLT) or a combination of the two.

Mo-LT contains two sub-sets, pure LT (Pu-LT) and auto-simultaneous LT (ASi-LT). Pu-LT describes a single reaction achieved with a single laser, for example pain attenuation with a GaAlAs diode laser therapy system for pure LLLT (Pu-LLLT) or precise, nonthermal corneal ablation with the eximer laser (Pu-HLLT).

ASi-LT is used to describe two or more simultaneous reactions achieved with the one laser, such as has been discussed already in Figure 1 above where the complete range of therapeutic and surgical reactions occur simultaneously, and includes protein denaturational LT (Pr-LT); hemocoagulational LT (H-LT); vaporizational

Table 1: Main subsets of laser treatment (LT)

\begin{tabular}{lc}
\hline \hline Type & Abbreviation \\
\hline Mono-type LT & Mo-LT \\
\hline Multi-type LT & Mu-LT \\
\hline Concomitant-type LT & Cc-LT \\
\hline \hline
\end{tabular}


Table 2: Mono-type laser treatment (Mo-LT) and its subsets

\begin{tabular}{clc}
\hline \hline \multicolumn{1}{c}{ Type } & \multicolumn{1}{c}{ Description } & Abbreviation \\
\hline 1 & Mono-type laser treatment & Mo-LT \\
\hline $1-1$ & Pure laser treatment & Pu-LT \\
\hline $1-2$ & $\begin{array}{l}\text { Autosimultaneous laser } \\
\text { treatment }\end{array}$ & ASi-LT $^{\dagger}$ \\
\hline $1-2-1$ & $\begin{array}{l}\text { Protein denaturational laser } \\
\text { treatment }\end{array}$ & ASi.Pr-LT \\
\hline $1-2-2$ & $\begin{array}{l}\text { Haemocoagulational laser } \\
\text { treatment }\end{array}$ & ASi.H-LT \\
\hline $1-2-3$ & $\begin{array}{l}\text { Vaporizational laser } \\
\text { treatment }\end{array}$ & ASi.V-LT \\
\hline $1-2-4$ & $\begin{array}{l}\text { Carbonizational laser } \\
\text { treatment }\end{array}$ & ASi.C-LT \\
\hline $1-2-5$ & $\begin{array}{l}\text { Miscellaneous others } \\
\text { † destructive or photoactivative: }\end{array}$ & $\begin{array}{c}\text { ASi.D-LT (HLLT) } \\
\text { ASi.A-LT (LLLT) }\end{array}$ \\
\hline \hline & &
\end{tabular}

LT (V-LT); carbonizational LT (C-LT) and other reactions such as photo-osmotic, photodynamic, photoelectric effects and so on. Any of these destructive type reactions will most often be combined with a biostimulative reaction at the outer periphery of the beam, due to the attenuation of the incident photon density as it penetrates the target tissue. Mono-type LT and its subsets are summarized in Table 2.

It is important to remember that the present study encompasses both laser therapy and laser surgery under the single heading of laser treatment (LT), and that the lasers may be used alone, in combination with themselves or with each other, depending on the classification type. The examples given, while only used hypothetically in this study, are drawn from the au-

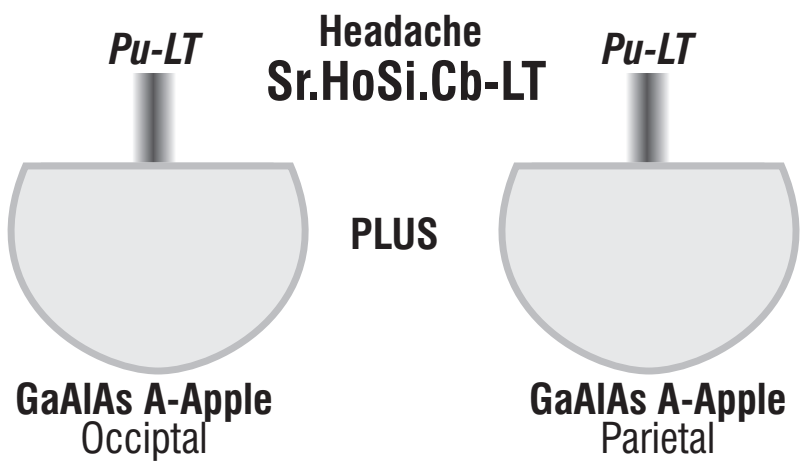

Fig 3: An example of same-reactive homo-simultaneous combined laser treatment, with the same laser being used on different sites (in this example GaAlAs A-Apples) in the same treatment session to achieve the same effect (in this example, simultaneous pain attenuation of the occiptial and parietal areas in a patient with headache). thor's experience and will be illustrated in subsequent clinically-based studies.

Type 2: Multi-type Laser Treatment (Mu-LT)

Mu-LT has two main sub-sets, combined LT (Cb-LT) and compound LT (Cp-LT). Each of these sub-sets contain homogeneous-combined and compound LT (HoCb-LT, HoCp-LT) and xenogeneous-combined and compound LT (XeCb-LT, XeCp-LT). The sub-types of combined laser treatment (Cb-LT) will now be discussed in detail, but the reader should bear in mind that they can be applied identically to compound laser treatment (Cp-LT). In the interests of space, Cp-LT will thus not be illustrated.

\section{Type 2-1: Combined LT (Cb-LT)}

Cb-LT describes treatment of the one disease existing in one or multiple sites with the same or different lasers, simultaneously or on successive applications or sessions. Type 2-1-1: Homogeneous Cb-LT

When the same lasers are used in combination, it is referred to as homogeneous Cb-LT, Ho.Cb-LT. If the same laser types are used at the same time, it is referred to as homo-simultaneous combined laser treatment, or HoSi.Cb-LT. In this case, the reaction produced by the lasers may be the same (same-reactive HoSi.Cb-LT, Sr.HoSi.Cb-LT), or they may produce different reactions (different-reactive HoSi.Cb-LT, Dr.HoSi.Cb-LT).

In a patient with severe headache, two GaAlAs diode lasers may be applied simultaneously (A-Apples), one to the occipital zone and the other to a point on the parietal zone: in both cases the reaction is the same, namely the relief from the headache. ${ }^{2}$ This is an example of same-reactive homo-simultaneous combined laser

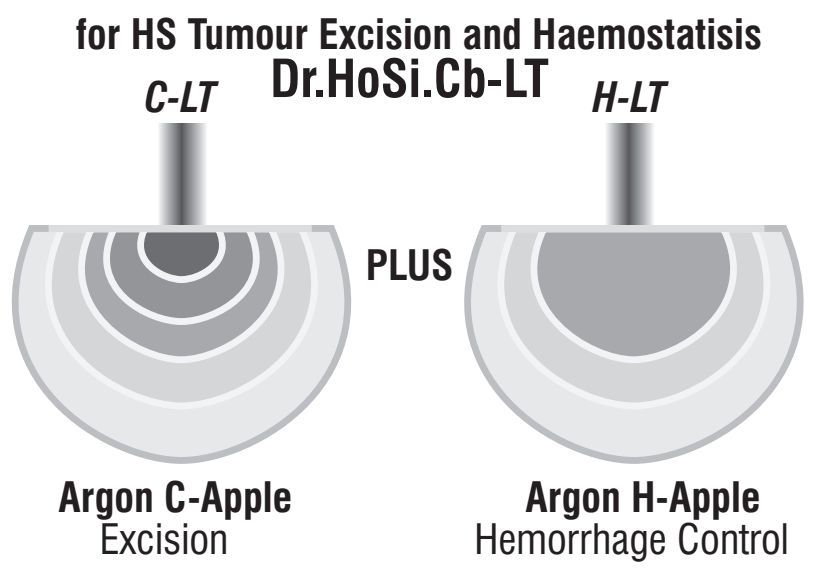

Fig 4: Two argon lasers are being used simultaneously, one in the focused mode for excision of a soft tumor in a hemangioma simplex lesion and the other in the defocused mode for hemocoagulation to control hemorrhage in the operative field. This is an example of different-reactive homo-simultaneous combined laser treatment, with the same lasers being used in the same operative field. 


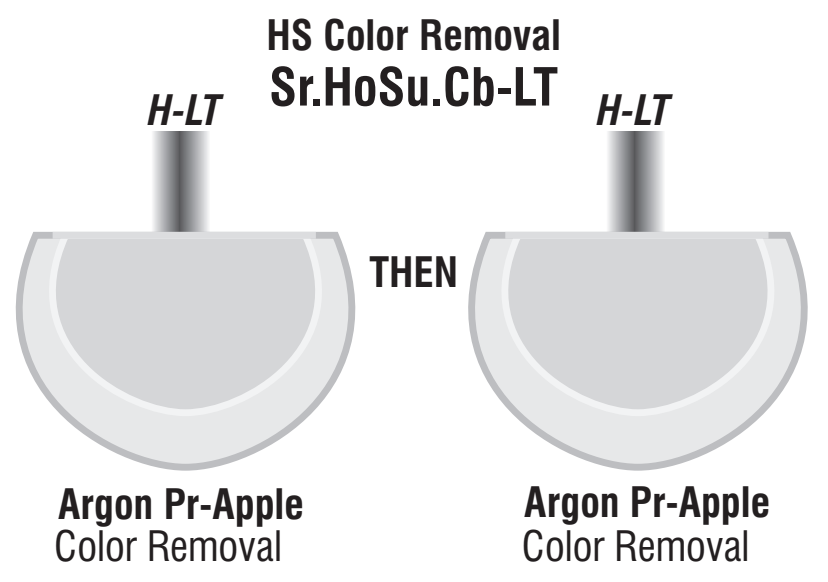

Fig 5: In same-reactive homo-successive combined laser treatment, the same laser is used on successive occasions to achieve the same effect such as staged color removal in the author's zebra method for treatment of hemangioma simplex using the argon laser in an H-Apple configuration.

treatment (Sr.HoSi.Cb-LT), and is illustrated in Figure 3.

In the second case, a soft tumor in a hemangioma simplex lesion is being excised with an argon laser using a highly focused beam (C-Apple), and at the same time the defocused beam of another argon system is used to control hemorrhage of vessels in the wound bed ( $\mathrm{H}-\mathrm{Ap}$ ple). This is different-reactive homo-simultaneous combined laser treatment (Sr.HoSi.Cb-LT) and is illustrated in Figure 4.

The same lasers may be applied at different times, rather than at the same time, either in the one treatment session or in different treatment sessions. This is referred to as homo-successive combined laser treatment, or HoSu.Cb-LT. Once again the reactions produced may be the same, (same-reactive HoSu.Cb-LT, Sr.HoSu.Cb-LT), or the reactions may differ (different-reactive HoSu.Cb-LT, Dr.HoSu.Cb-LT).

A patient has haemangioma simplex existing at several tissue layers. The defocused argon laser is used in Ohshiro's linear zebra method ${ }^{7}$ to remove the colour in protein denaturation ( $\mathrm{Pr}$-Apple), leaving linear strips of untreated tissue between the treated areas. After the treated area has successfully reepthelialized, the untreated areas are treated in the same manner with the argon laser. This is same-reactive homo-successive combined laser treatment (Sr.HoSu.Cb-LT), and is illustrated in Figure 5.

The focused $\mathrm{CO}_{2}$ laser is used to excise a large verruca vulgaris (C-Apple). Some small points of hemorrhage are seen in the wound bed, and are controlled with the defocused $\mathrm{CO}_{2}$ laser ( $\mathrm{H}$-Apple). The same laser is then completely defocused and the area of the wound is 'painted' with the $\mathrm{CO}_{2}$ beam at a very low incident power density for wound healing and viral inactivation (A-Apple). This is different-reactive homo-successive combined laser treatment (Dr.HoSu.Cb-LT), and is illustrated in Figure 6.

Type 2.1.2: xenogeneous Cb-LT

Rather than the same laser type, different laser types may also be combined in laser treatment of the one disease, known as xenogeneous Cb-LT, Xe.Cb-LT. Xe.Cb-LT has the same sub-types as Ho.Cb-LT already discussed above. The following are representative exam-

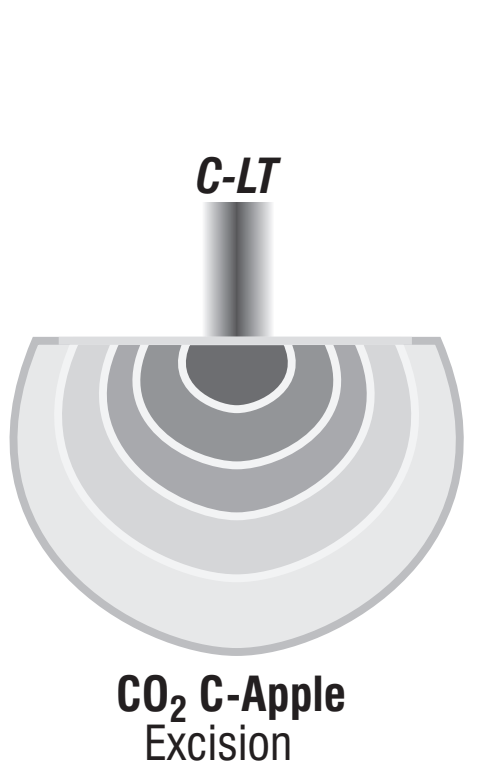

\section{Verruca Excision, Hemostasis and LLLT Dr.HoSu.Cb-LT}
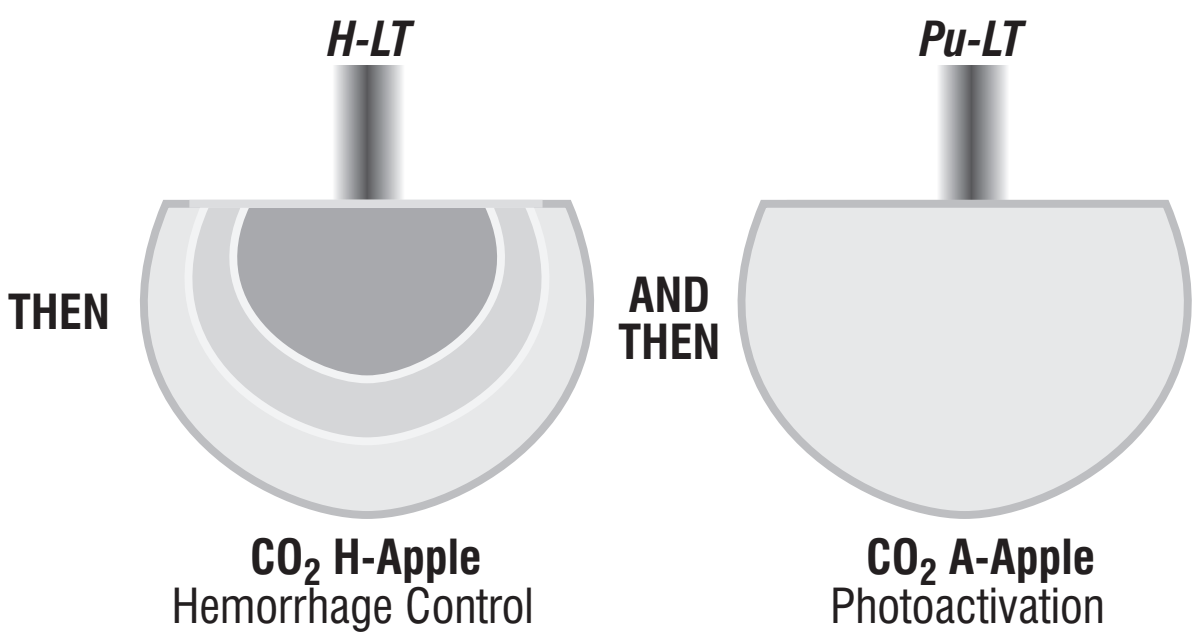

Fig 6: Focused $\mathrm{CO}_{2} \mathrm{C}$-Apple is used to excise a verruca vulgaris followed by defocused $\mathrm{CO}_{2} \mathrm{H}-\mathrm{Apple}_{\mathrm{p}}$ in hemostatic control of the wound bed. Finally $\mathrm{CO}_{2}$ LLLT is applied for accelerated wound healing and viral inactivation. This is different reactive homo-successive combined laser treatment. 


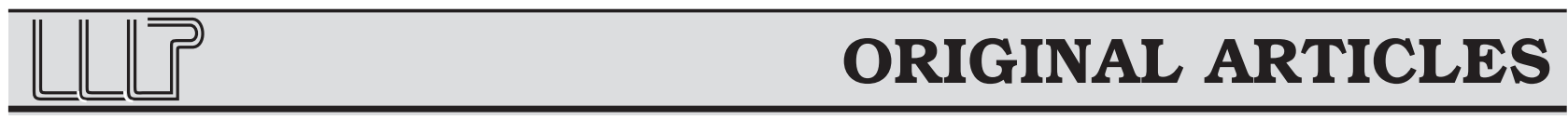

a:

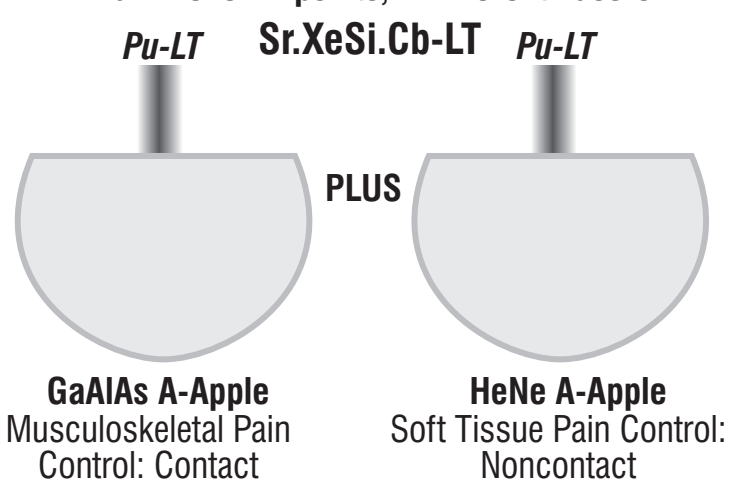

b:

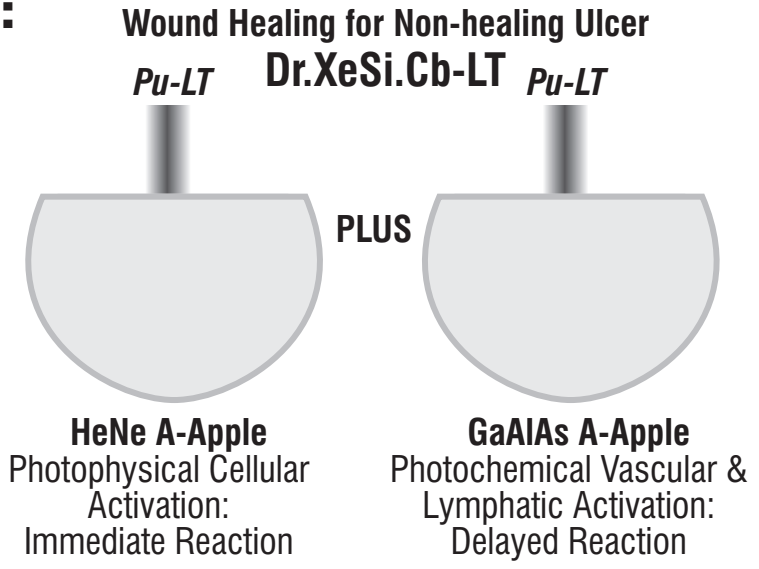

Fig 7: a: GaAlAs LLLT is applied for deeper-seated musculoskeletal pain in a patient's neck while at the same time the $\mathrm{HeNe}$ laser is applied over the larynx to relieve the pain in that tissue. This is an example of same-reactive xeno-simultaneous combined laser treatment. b: an example of different-reactive xeno-simultaneous combined laser treatment in the treatment of a chronic crural ulcer. HeNe LLLT is applied to the surface of the ulcer in the scanning non-contact mode for superficial photochemical cellular activation and the GaAlAs laser is applied in contact mode around the periphery of the ulcer to activate photophysically the deeper lymphatic and blood vessel systems.

ples.

In a patient with severe whiplash injury, GaAlAs LLLT is applied to the deeper-seated musculoskeletal pain in the neck while at the same time the HeNe laser is applied in the noncontact mode to the oropharynx to relieve the pain in that tissue. This is an example of same-reactive xeno-simultaneous combined laser treatment (Sr.XeSi.Cb-LT), and is illustrated in Figure 7a. In figure $7 \mathrm{~b}$, an example of different-reactive xeno-simultaneous combined laser treatment (Dr.XeSi.Cb-LT) is illustrated in the treatment of a chronic crural ulcer. HeNe LLLT is applied to the surface of the ulcer in the scanning non-contact mode to modulate the activity of the superficial cellular components of the ulcer (photochemical action), while the GaAlAs laser is applied in a:

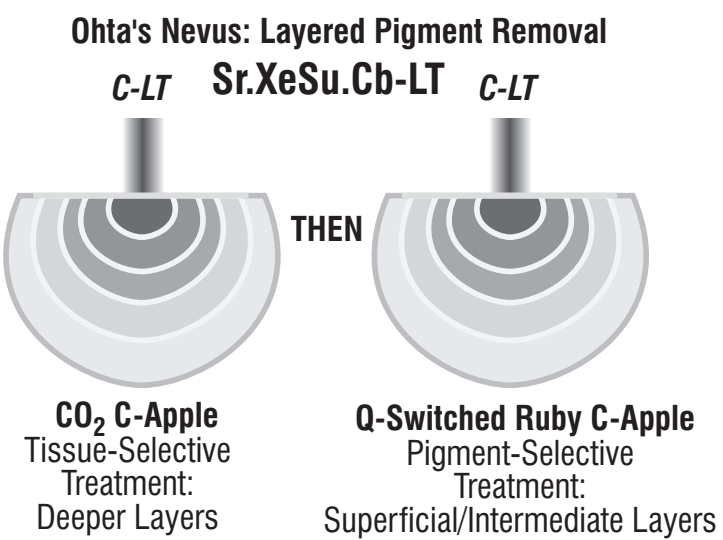

b:

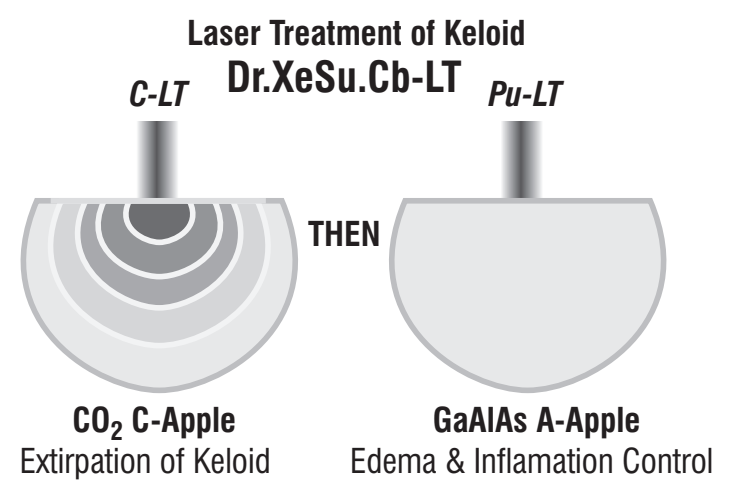

Fig 8: a: Same-reactive xeno-successive combined laser treatment being used in tissue-selective and pigment-selective photothermal mechanisms: the focused $\mathrm{CO}_{2}$ laser is used in the spotty fashion to remove Ohta's nevus pigment in the dermis. The Q-switched ruby is later used to remove remaining pigment in the deeper areas. b: A $\mathrm{CO}_{2}$ laser is used to excise a keloid and GaAlAs LLLT is applied to the borders of the excision to control postoperative edema, to modulate the inflammatory response and as prophylaxis against scarring in different-reactive xeno-successive combined laser treatment.

contact mode around the periphery of the ulcer to activate the deeper lymphatic and blood vessel systems (photophysical action).

In the treatment of Ohta's nevus using same-reactive xeno-successive combined laser treatment (Sr.XeSu.Cb-LT), the focused $\mathrm{CO}_{2}$ laser is used in the spotty fashion to remove the melanin pigment in the deep dermis. ${ }^{7}$ Following successful and complete healing, the Q-switched ruby is used to remove remaining pigment in the superficial to intermediate layers of the skin. Both systems rely on a pigment-selective photothermal mechanism (Figure 8a). Following excision of a keloid with the focused $\mathrm{CO}_{2}$ laser and suturing of the wound, GaAlAs LLLT is applied to the borders of the excision to control postoperative edema, to modulate the inflammatory response and as prophylaxis against scarring in different-reactive xeno-successive combined laser treatment (Dr.XeSu.Cb-LT, Figure 8b). 
Table 3: Summarized classification of Multi-type laser

\begin{tabular}{|c|c|c|}
\hline Type & Classification & Abbreviation \\
\hline 2: & Multi-type LT & Mu-LT \\
\hline 2-1: & Combined LT & $\mathrm{Cb}-\mathrm{LT}$ \\
\hline 2-1-1: & Homogeneous Cb-LT & Ho.Cb-LT \\
\hline 2-1-1-a: & Homo-simultaneous Cb-LT & HoSi.Cb-LT \\
\hline 2-1-1-a-i: & Same reactive HoSi.Cb-LT & Sr.HoSi.Cb-LT \\
\hline 2-1-1-a-ii: & Different reactive HoSi.Cb-LT & Dr.HoSi.Cb-LT \\
\hline $2-1-1-b:$ & Homo-successive Cb-LT & HoSu.Cb-LT \\
\hline 2-1-1-b-i: & Same reactive HoSu.Cb-LT & Sr.HoSui.Cb-LT \\
\hline 2-1-1-b-ii: & Different reactive HoSu.Cb-LT & Dr.HoSu.Cb-LT \\
\hline 2-1-2: & Xenogeneous $\mathrm{Cb}-\mathrm{LT}$ & $\mathrm{Xe} \cdot \mathrm{Cb}-\mathrm{LT}$ \\
\hline 2-1-2-a: & Xeno-simultaneous Cb-LT & XeSi.Cb-LT \\
\hline 2-1-2-a-i: & Same reactive XeSi.Cb-LT & Sr.XeSi.Cb-LT \\
\hline 2-1-2-a-ii: & Different reactive XeSi.Cb-LT & Dr.XeSi.Cb-LT \\
\hline 2-1-2-b: & Xeno-successive $\mathrm{Cb}$-LT & XeSu.Cb-LT \\
\hline 2-1-2-b-i: & Same reactive XeSu.Cb-LT & Sr.XeSui.Cb-LT \\
\hline 2-1-2-b-ii: & Different reactive XeSu.Cb-LT & Dr.XeSu.Cb-LT \\
\hline 2-2: & Compound LT (Same classifications as Cb-LT) & Cp-LT \\
\hline
\end{tabular}

\section{Type 2-2: Compound Laser Treatment (Cp-LT)}

In $\mathrm{Cp}-\mathrm{LT}$ the same subdivisions exist as for combined laser treatment, the difference being that in Cp-LT two or more diseases are being treated with the same type of laser (HoCp-LT) or different laser types (XeCp-LT), with exactly the same sub-types as for Cb-LT. Table 3 summarizes the classification of the multi-type of laser treatment (Mu-LT).

Type 3: Concomitant LT (Cc-LT)

Each disease is different, and each patient is an individual; furthermore the same disease may exist in different stages or degrees on the one patient site-by site. Accordingly the author has developed over the last 20 years his Total Treatment Concept, which calls for the flexible design of a treatment protocol based on each patient's individual needs. This concept requires the use of all of the LT types already discussed and where necessary used concomitantly with conventional treatment methods, such as snowy dry ice followed by epithelial peeling for the superficial pigment in Ohta's nevus, followed by the focused argon laser for remaining patches of deeper- situated nevus cells which have not responded to the snowy dry ice/epithelial peeling regime; and finishing with the focused Q-switched ruby or Nd:YAG lasers for the re- maining superficial and intermediate scattered pigment. The GaAlAs diode laser would be used during each session: during the snowy dry ice/epithelial peeling for pain attenuation, and following the laser sessions to ensure good wound healing and minimize unwanted side effects.

\section{Discussion}

The question of the correct use of terminology and its importance in the accurate reporting of clinical and surgical trials and experiments has been dealt with in some detail in the literature. ${ }^{(8-10)}$ Without accurate reporting and appropriate terminology other researchers are unable accurately to repeat clinical trials or experimental studies to corroborate data and validate findings. This leads to lack of scientific acceptance of the specific study or trial in particular and of the laser in surgery and medicine in general. Therapeutic applications of the laser tend to have more problems along these lines than surgical applications, mostly because laser surgery has a much longer history and is used in many more specialities than is the case with laser therapy. An example of current terminology which lacks a scientific base is the popular use of such terms as 'low power laser' and 'high 

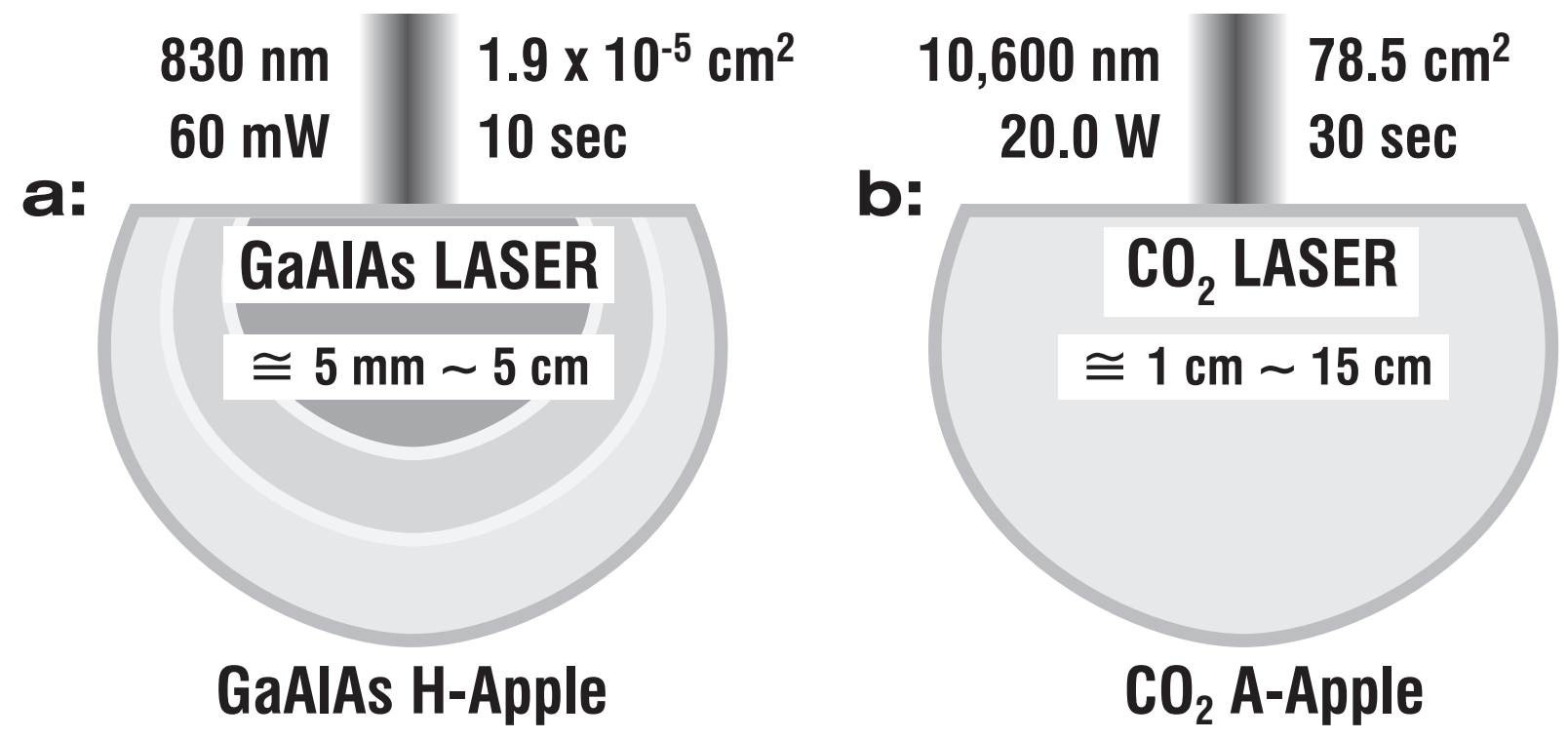

Fig 9: Laser Apples illustrating the fallacy behind 'low power laser'. a: A GaAlAs diode laser with a very low output power normally associated with activation apple (A-Apple) patterns is capable, with appropriate parameters, of creating an H-Apple pattern in the destructive apple (D-Apple) group: b: This $\mathrm{CO}_{2}$ laser with a comparatively high output power, normally associated with destructive (D-Apple) patterns, creates an A-Apple pattern. Thus the terms 'high power laser' and 'low-power laser' are scientifically inaccurate and should be avoided in discussions regarding clinical laser applications (from ref. 4, used with permission).

power laser', or the marketing-driven and even more unscientific 'soft laser' and 'hard laser' to refer to therapeutic laser systems and surgical laser systems, respectively. The author is of the opinion that while there may be lasers with low output powers and lasers with high output powers, they can actually both be used to produce an entire range of tissue reactions.

The most important point the author wishes to stress is that the tissue reaction should be used to classify laser systems, and not any aspect of the hardware. A laser is merely the device which is capable of generating a beam of laser energy. It is the reaction with the tissue which gives this beam of special energy its clinical utility, whether for laser surgery or for laser therapy. Figure 8 illustrates the use of a 'high power laser' to produce a therapeutic level dosage and a so-called 'low-power laser' delivering potentially photodestructive incident power densities. ${ }^{4}$ That is why the author has developed his classification system based on the tissue reaction, and has also designed the 'laser apple' concept so that a single pictorial representation is capable of giving all salient characteristics of a laser beam which not only includes all the physical parameters associated with the laser itself but also goes further and graphically represents the laser tissue reactions and the beam spread and penetration. When the laser apple is coupled with the author's classification criteria, the laser clinician can report in one simple diagram the entire range of parame- ters necessary for other colleagues to repeat the work.

\section{References}

1: Ohshiro T, and Calderhead RG (1988): Low Level Laser Therapy: A Practical Introduction. John Wiley \& Sons, Chichester, UK.

2: Ohshiro T (1991): Low Reactive Level Laser Therapy: Practical Application. John Wiley \& Sons, Chichester, UK.

3: Ohshiro T (1995): Laser Treatment for Naevi. John Wiley \& Sons, Chichester, UK.

4: Ohshiro T (1996): The laser apple: a new graphic representation of medical laser applications. Laser Therapy, 8: $185-190$

5: Kato K, and Nagasawa A (1991): Imaging technique for near infrared GaALAs diode laser beam distribution in tissue. Laser Therapy, 3: 67-70.

6: Nagasawa A: (1991): Applications of LLLT in dentistry. In Ohshiro T: Low Reactive-level Laser Therapy: Practical Application. John Wiley \& Sons, Chichester, UK. pp 76-98.

7: Ohshiro T (1995): Laser treatment. In Ohshiro T: "Laser Treatment for Naevi." John Wiley \& Sons, Chichester, UK. Pp 171-201.

8: Ohshiro T, and Calderhead RG: Preface. In Low Level Laser Therapy: A Practical Introduction. John Wiley \& Sons, Chichester, UK. Pp xi-xii.

9: Ohshiro T (1989): Editorial: On Parameters. Laser Therapy, 1: $117-118$.

10: Calderhead RG (1991): Watts a joule: on the importance of accurate and correct reporting of laser parameters in low reactive-level laser therapy and photobioactivation research. Laser Therapy, 3: 177-182. 